LONDOÑO, Fernando; MALDONADO, Francisco, "Ochenta artículos para

celebrar ochenta años: el Instituto de Ciencias Penales y los “clásicos" de su Revista de

Ciencias Penales'

Polít. crim. Vol. 14, No 27 (Julio 2019), Doc. 1, pp. 599-605

[http://politcrim.com/wp-content/uploads/2019/06/Vol14N27D1.pdf]

\title{
Ochenta artículos para celebrar ochenta años: el Instituto de Ciencias Penales y los "clásicos" de su Revista de Ciencias Penales*
} Eighty articles to celebrate eighty years: the Instituto de Ciencias Penales and the
"classics" of its Revista de Ciencias Penales

Dr. Fernando Londoño Martínez

Profesor Asociado de la Universidad Diego Portales

Ex Secretario Ejecutivo del Instituto de Ciencias Penales (2013-2017)

fernando.londono@udp.cl

Dr. Francisco Maldonado Fuentes

Director del Centro de Estudios de Derecho Penal

Profesor de Derecho Penal de la Universidad de Talca

Ex Secretario Ejecutivo del Instituto de Ciencias Penales (2011-2013)

franciscomaldonado.2006@gmail.com

\section{Resumen}

El texto que aquí se reproduce corresponde en lo fundamental a la presentación de un libro reciente (Clásicos de la literatura penal en Chile. La Revista de Ciencias Penales en el siglo XX: 1935-1995, 2 tomos, Valencia: Tirant Lo Blanch, 2018), cuya edición fue encargada a los autores de este documento por el Instituto de Ciencias Penales. En aquel libro se republica una selección de ochenta artículos significativos de la Revista de Ciencias Penales, publicados originalmente entre 1935 y 1995. Junto con ofrecer detalles sobre el contexto y las personas que hicieron posible el libro, la presentación sirve de algún modo para rendir un homenaje a la dogmática chilena, así como para renovar la conmemoración de los ochenta años del Instituto.

Palabras clave: Revista de Ciencias Penales, Instituto de Ciencias Penales, derecho penal chileno, dogmática penal.

\begin{abstract}
The following text is basically the presentation of a recent book (Clásicos de la literatura penal en Chile. La Revista de Ciencias Penales en el siglo XX: 1935-1995, 2 tomos, Valencia: Tirant Lo Blanch, 2018), whose edition was entrusted to the authors by the Instituto de Ciencias Penales. The book thus presented consists of a selection of eighty significant articles originally published in the Revista de Ciencias Penales, between 1935 and 1995. Along with giving details about the context and the people to whom the authors are grateful for the book, the presentation is also a way of paying homage to the Chilean criminal law doctrine, as well as to renew the commemoration of the Institute's eighty years since its foundation in 1937.
\end{abstract}

Key words: Revista de Ciencias Penales, Instituto de Ciencias Penales, Chilean criminal law, criminal law doctrine. 
La obra que hoy se presenta $^{1}$ - una colección de ochenta artículos de los primeros sesenta años de vida de la Revista de Ciencias Penales (en adelante Clásicos) - sólo se explica a partir de un esfuerzo mancomunado, en un sentido muy fuerte del término, al modo de una carrera de relevos. Dos dimensiones constituyen el espacio de ese relevo. Primeramente, el material aquí publicado es fruto del trabajo de tres generaciones de autores y editores, activas en Chile entre 1935 y 1995. Luego, la selección y edición necesaria para estos volúmenes se debe a tres directorios sucesivos del Instituto de Ciencias Penales. Los lazos que han posibilitado esta publicación son entonces intergeneracionales e institucionales.

Seguramente serán muy leídas las reseñas que encabezan los volúmenes - preparadas por Luis Ortiz ${ }^{2}$ y por Juan Rauld ${ }^{3}$ — de modo que allí podrán encontrar los lectores respuesta a la pregunta por el locus de aquellos lazos: es decir, la rica y coral historia del Instituto y de la Revista, que es en parte la historia de la penalística chilena. Por eso, aquí bastará con decir que esa historia explica este presente: y es que, si nuestra dogmática penal tiene tan buen presente, se debe a la laboriosa generosidad de estudiosos empeñados en el siglo XX, a varios de los cuales se honra con la reedición de sus trabajos. A ese acervo - ofrecido por escrito, pero que no descarta el menos tangible de la entrega oral - es a lo que llamamos tradición. Con ella, desde ella - en su canon arranca el empeño-crítico personal y dialógico, con ganancia para la tradición y para quienes se hacen de ella.

En este contexto - es decir, apropósito del quehacer de nuestra tradición dogmática bien valga un excurso para dejar hablar a Enrique Cury, quien en 1964 ofrecía un balance sobre la evolución de la dogmática penal chilena, a la vez que un "programa de acción". El texto corresponde precisamente a uno de los artículos republicados en los Clásicos, y la cita que a continuación leerán arranca desde un pasaje en el que Enrique Cury lamenta el abandono que por entonces afectaba a nuestra Parte Especial ${ }^{4}$, desarzonada de la más elaborada y redonda Parte General, con desprestigio no tanto (o no sólo) para la Parte Especial, sino para la propia Parte General, en la medida que no

*El texto aquí publicado corresponde en lo fundamental a lo leído por Fernando Londoño en la presentación de los volúmenes indicados en la nota $\mathrm{n}^{\circ} 1$ - Clásicos - en el marco de las XV Jornadas de Derecho Penal y Ciencias Penales, U. Alberto Hurtado, el 21 de noviembre de 2018. Materialmente, se trata de una ampliación y adaptación acometida por Fernando Londoño en base a la nota editorial con la que se encabezan aquellos volúmenes, firmada por quienes suscribimos la presente contribución.

${ }^{1}$ Se trata de LONDOÑO, Fernando; MALDONADO, Francisco (Eds.), Clásicos de la literatura penal en Chile. La Revista de Ciencias Penales en el siglo XX: 1935-1995, 2 tomos, Valencia: Tirant Lo Blanch, 2018. La edición de esa obra le fue confiada por el Instituto de Ciencias Penales a los aludidos editores, en su calidad de ex Secretarios Ejecutivos de aquella corporación. Quienes suscribimos este documento expresamos nuestra gratitud al Instituto por aquella confianza.

2 ORTIZ, Luis, "Presentación”, en: Londoño, Fernando; Maldonado, Francisco (Eds.), Clásicos de la literatura penal en Chile. La Revista de Ciencias Penales en el siglo XX: 1935-1995, 2 tomos, Valencia: Tirant Lo Blanch, 2018, pp. 23-26.

${ }^{3}$ RAULD, Juan, "Ocho décadas de la Revista de Ciencias Penales y del Instituto de Ciencias Penales. 1935-2015: hitos y testimonios”, en Londoño, Fernando; Maldonado, Francisco (Eds.), Clásicos de la literatura penal en Chile. La Revista de Ciencias Penales en el siglo XX: 1935-1995, 2 tomos, Valencia: Tirant Lo Blanch, 2018, pp. 27-50.

${ }^{4}$ El lamento no podría justificarse del todo hoy, a nuestro parecer, considerada la atención que en los últimos treinta o cuarenta años ha recibido la Parte Especial por nuevas generaciones de penalistas chilenos, en muchos casos con densa conciencia del sentido operativo de la Parte General. Con todo, que bien entrado el siglo XXI no exista en nuestro medio un Comentario para la Parte Especial, es algo que habla por sí mismo sobre las limitaciones en este plano. 
lograba probar su rendimiento allí donde más cuenta para la praxis. ¿Qué hacer?, se pregunta Enrique Cury.

\section{Ahora su respuesta:}

"Ciertamente a "grandes males corresponden grandes remedios" (...) "Lo primero parece ser reiniciar la tarea con humildad. Algunos de nuestros juristas pueden ser tan agudos y profundos como Mezger, Welzel o Maurach, pero es preciso reconocer que el campo en que trabajamos no se encuentra todavía suficientemente preparado para la estructuración de sistemas dogmáticos tan completos y cerrados como los de esos tratadistas. Primero tenemos que encontrar nuestra ley positiva y, luego, tenemos que aceptarla." (...)

Luego es menester intentar el reanálisis de nuestras instituciones penales, comenzando, en lo posible, por las de la Parte Especial. (...) Esa tarea tiene que generalizarse y convertirse en preocupación fundamental de cada jurista en la medida de sus posibilidades."

A propósito de la dedicación a la Parte Especial, sigue Enrique Cury con una sugerencia metodológica (y sustantiva):

"Es necesario comprender, claro está, que los grandes sistemas extranjeros contienen bases estructurales valiosísimas. Sería absurdo desaprovecharlas. Pero no debemos renunciar al análisis minucioso de cada una de ellas, sometiéndolas a prueba en la continua comparación con nuestros preceptos legales positivos y rechazándolas sin vacilación, por perfectas, simétricas o estéticas que parezcan, tan pronto lleguemos a la convicción de que no son aceptadas por la ley o carecen de fundamento en ella."6

Y remata con un tercer consejo, aparentemente contradictorio con el primero, en el que sugería humildad:

"Por último, es necesario no perder jamás de vista la Teoría General del Derecho. Aparentemente esta pretensión es contradictoria con la de humildad a que aludíamos antes. Nada más equivocado. Por el contrario, el desarrollo auspicioso de los estudios penales en las últimas décadas se ha constituido en una peligrosa tentación de vanidad para los juristas. Es menester desecharla, teniendo en consideración que ese desarrollo y su importancia derivan, no de que operemos con un objeto de conocimiento más o menos importante que el de cualquier otra rama de la ciencia jurídica sino, precisamente, de que ese objeto ha sido estudiado con mayor rigor en los últimos tiempos, haciendo así aportaciones valiosas a la teoría general. Por lo mismo es preciso tener la vista puesta sobre la totalidad del ordenamiento jurídico en su innegable unidad."

Con eso se cierra el excurso. Mucho daría la cita para discutir, pero no puede ser esta la ocasión, naturalmente.

\footnotetext{
5 CURY, Enrique, "Reflexiones sobre la evolución del derecho penal chileno", Revista de Ciencias Penales, t. XXIII (1964), pp. 154 - 160, pp. 159-160; ahora también en Londoño, Fernando; Maldonado, Francisco (Eds.), Clásicos de la literatura penal en Chile. La Revista de Ciencias Penales en el siglo XX: 1935-1995, 2 tomos, Valencia: Tirant Lo Blanch, 2018, t. II, pp. 1103-1109, pp. 1108-1109.

${ }^{6}$ CURY, "Reflexiones", cit. nota n5, p. 159 / p. 1109.

${ }^{7}$ CURY, "Reflexiones", cit. nota n5, pp. 159-160/p. 1109.
} 
Se decía pues que la historia de la Revista y del Instituto es conocida (por los mayores) y los más jóvenes seguiremos aprendiendo sobre ella. Aquí sólo toca explicar brevemente la génesis de la obra Clásicos y ofrecer los muchos agradecimientos debidos.

Pues bien, teniendo como marco la producción doctrinaria de los primeros sesenta años de la Revista, la selección de los artículos la realizó el directorio del Instituto entre 2012 y 2013. Esta selección se desarrolló en tres etapas. En la primera de ellas se levantó el listado completo de la colección de la Revista, labor a cargo de Carlos González, becario de la Universidad de Talca. Sobre dicho listado, y con el objeto de generar una propuesta preliminar de los textos seleccionables, se procedió a subdividir la colección, encomendándose una revisión detallada del contenido de los diversos artículos a algunos integrantes de aquel directorio. Concretamente a Magdalena Ossandón, Raúl Carnevali, Héctor Hernández y Juan Ignacio Piña, además de uno de quienes firman esta contribución (Francisco Maldonado), por entonces secretario ejecutivo del Instituto.

Para realizar dicha selección se tuvo en cuenta que la Revista aglutina la evolución del pensamiento jurídico penal chileno propio de su época (1935 a 1995). Se procuró por ello privilegiar la consideración de las obras de dogmática jurídico-penal, por sobre los trabajos de criminología, criminalística o política criminal, en atención a que estos últimos se concentraron preferentemente en cuestiones contingentes (una muestra de aquellos existe, en todo caso y puede apreciarse fácilmente consultando el índice por materias). Se priorizaron asimismo las obras de autores nacionales que hubieren tenido impacto en el desarrollo de la ciencia jurídico-penal chilena, con total independencia de su vigencia actual o su dedicación a tópicos que pueden hoy considerarse parcialmente superados. Finalmente, se concedió una especial atención a aquellas obras que no hubieren recibido mayor difusión en otros medios.

Formulada una primera propuesta, los directores del Instituto, Alfredo Etcheberry, Luis Ortiz, Carlos Künsemüller y Jaime Campos, procedieron a su revisión con base en el listado total de artículos que comprende la colección, sugiriendo las modificaciones que consideraron oportunas a partir de los mismos criterios orientadores. Quedó así configurado el grueso del material, sin perjuicio de puntuales sustracciones posteriores, conforme a criterios aplicados por los editores.

El testigo del relevo fue asumido por los siguientes dos directorios del Instituto, ambos presididos por Luis Ortiz y con otro de los que firma esta nota (Fernando Londoño) en calidad de secretario ejecutivo. Se trató aquí de llevar a término la labor de conversión de los textos en un formato manejable para su re-publicación. De este modo, los textos digitales fueron revisados detalladamente, confrontándolos con los originales. En ese contexto, se insertaron entre corchetes [ ] referencias específicas a la numeración de la página original, de modo que el material publicado en estos volúmenes pueda citarse conforme al texto original de la Revista, introduciéndose en cada caso una nota al pie con dichas coordenadas. En lo demás, se procuró dejar perfectamente intactos los textos. Se incorporaron, sin embargo, breves notas aclaratorias, en los contados casos en 
los que una errata del texto original pudiere justificarlo o allí donde los editores consideraron que un cambio meramente formal se justificaba ${ }^{8}$.

En esta fase fue decisiva la colaboración de un grupo de ayudantes del Departamento de Derecho Penal de la Universidad Diego Portales, a quienes más abajo se mencionará en forma individual.

Paralelamente, la elaboración de una reseña dedicada a la historia de la Revista y del Instituto fue comisionada por el directorio a Juan Rauld, periodista y editor de la Revista Mensaje. Ya podrán apreciar los lectores la riqueza de aquella contribución. Juan Rauld se sumergió en las fuentes, primarias y secundarias, recogiendo además testimonios de actores de la época (María Inés Horvitz, Carlos Künsemüller, Jorge Mera, Luis Ortiz y Miguel Soto). Todo se volcó en un texto tan riguroso como animado. La nota histórica es precedida - y hace juego - con la Presentación de los volúmenes, a cargo de Luis Ortiz. Como verán, se trata de la adaptación de un texto que preparó Luis Ortiz para nuestras XI Jornadas en noviembre de 2014, contexto en el que se lanzaba la Sexta Época de la Revista. Un texto comprensivo, con notas vivas en la memoria de su autor, y que llevan el sello de lo concreto-real: como cuando se evoca la disputa dogmática entre Luis Cousiño, Alfredo Etcheberry y Eduardo Novoa Monreal, en el quinto piso de Huérfanos 1141.

Se decía que la nota de Luis Ortiz es de fines de 2014. ¿Cómo es que la publicación de los Clásicos tardó tanto?

Dificultades y cambios en la casa editorial que originalmente había comprometido la publicación retrasaron considerablemente la concreción del proyecto. Pero no hay mal que por bien no venga. Primero, porque el retraso acercó la publicación del libro a la celebración de los ochenta años del Instituto, celebrado recién el año pasado. Algo casual, quizás, pero cargado de simbolismo, cuanto más si la selección cerraba exactamente en 80 artículos (y sin que de modo alguno se haya tenido ese número en el horizonte desde el comienzo). Segundo, porque gracias a la eficaz mediación de Jean Pierre Matus (por entonces uno de los directores del Instituto), el profesionalismo de Laura Barrios (directora internacional de la editorial encargada de estos volúmenes) y la solvencia de Tirant lo Blanch, el proyecto se encaminó hasta concretarse, en papel y en formato electrónico.

Lo que resta es agradecer a quienes colaboraron en el último relevo de esta posta, allí donde los tiempos se estrechan: por su decisiva colaboración en la revisión de las pruebas de imprenta, se deben agradecimientos especiales a Claudio Arellano y Miguel

\footnotetext{
${ }^{8}$ Dos ejemplos de enmiendas formales pueden ofrecerse aquí, rápidamente, a modo de ilustración. Un primer caso atañe a ORTIZ, Luis, "Algunas consideraciones sobre la teoría de la acción finalista", texto que fuera publicado en 1965 en el t. XXIV de la Revista de Ciencias Penales, en dos partes (pp. 4-24 y pp. 101-120), que indudablemente constituían una unidad. Esa unidad se rompió sin embargo al no seguirse el orden secuencial de las notas en la segunda parte. La unidad se recompone en la edición contemporánea que aquí se presenta, dejando una doble numeración para las notas: la original y la corregida, secuencial. Otro ejemplo es el del nombre del autor Eduardo Brücher (BRÜCHER, Eduardo; ZAMORANO, Manuel, "Estudio Psicológico y Psiquiátrico sobre un centenar de delincuentes chilenos"), que aparece sin Umlaut (= Brucher) en el original de la Revista de Ciencia Penales de 1942 (t. VI, pp. 514). Los editores juzgamos adecuado corregir aquello en la edición contemporánea, devolviéndole al apellido su forma alemana.
} 
Garcés, de la Universidad de Talca, y de la Universidad Diego Portales a Josefa Bejarano, Víctor Beltrán, Jaime Cerda, Carlos Donoso, Javier Escobar, Bernardita Figueroa, Josefina Guzmán, Nicolás Núñez, Catalina Valdés y Juan Ignacio Vásquez. Algunos de ellos estuvieron además desde el comienzo en el trabajo de inserción de la paginación original y en la primera revisión de los textos escaneados (Jaime Cerda, Javier Escobar y Nicolás Núñez, a ellos un agradecimiento muy especial). Un reconocimiento también afectuoso es debido al profesor Javier Wilenmann, secretario ejecutivo del Instituto a partir de 2017, por su colaboración en algunos aspectos operativos durante la última etapa.

Se cierra esta nota con una cita de José Ortega y Gasset, cuyo vitalismo encuadra muy bien el propósito de toda re-publicación de un clásico, en su propio contexto. El hallazgo de la cita se debe a Michael Pawlik, quien precisamente la usa con propósitos similares ${ }^{9}$. Escribe el filósofo español:

"No hay más que una manera de salvar al clásico: usando de él sin miramiento para nuestra propia salvación, es decir, prescindiendo de su clasicismo, trayéndolo hasta nosotros, contemporaneizándolo, inyectándole pulso nuevo con la sangre de nuestras venas, cuyos ingredientes son nuestras pasiones y nuestros problemas. En vez de hacernos centenarios en el centenario, intentar la resurrección del clásico resumergiéndolo en la existencia." 10

De modo que "redescubiertos" estos clásicos de la literatura penal chilena, a nosotros espera la tarea más difícil: ¡contemporaneizarlos! Que los textos aquí reunidos puedan todavía dialogar con las dificultades de la dogmática-penal del siglo XXI, es algo que depende de los lectores, presentes y futuros. Si ello acontece, se prolongará el vínculo intergeneracional que ha constituido materia y resorte de esta obra y - antes que nada del Instituto, desde su fundación hace ochenta años. Ochenta trabajos, para ochenta años. Y que sean muchos más.

¡Larga vida al Instituto de Ciencias Penales!

\footnotetext{
9 PAWLIK, Michael, La libertad institucionalizada. Estudios de Filosofía jurídica y Derecho penal, Madrid: Marcial Pons, 2010, p. 7.

${ }^{10}$ ORTEGA Y GASSET, José, "Pidiendo un Goethe desde dentro. Carta a un alemán", en: Del mismo autor, Obras Completas, t. IV (1929-1933), 5ª ed., Madrid: Revista de Occidente, 1962, pp. 395-420, p. 419; original publicado en abril de 1932.
} 


\section{Bibliografía}

BRÜCHER, Eduardo; ZAMORANO, Manuel, "Estudio Psicológico y Psiquiátrico sobre un centenar de delincuentes chilenos", en Revista de Ciencia Penales, t. VI (1942), pp. 5-14; republicado en Londoño, Fernando; Maldonado, Francisco (Eds.), Clásicos de la literatura penal en Chile. La Revista de Ciencias Penales en el siglo XX: 1935-1995, 2 tomos, Valencia: Tirant Lo Blanch, 2018, t. I, pp. 319-330.

CURY, Enrique, "Reflexiones sobre la evolución del derecho penal chileno", en Revista de Ciencias Penales, t. XXIII (1964), pp. 154 - 160; republicado en Londoño, Fernando; Maldonado, Francisco (Eds.), Clásicos de la literatura penal en Chile. La Revista de Ciencias Penales en el siglo XX: 1935-1995, 2 tomos, Valencia: Tirant Lo Blanch, 2018, t. II, pp. 1103-1109.

LONDOÑO, Fernando; MALDONADO, Francisco (Editores), Clásicos de la literatura penal en Chile. La Revista de Ciencias Penales en el siglo XX: 1935-1995, 2 tomos, Valencia: Tirant Lo Blanch, 2018.

ORTEGA Y GASSET, José, "Pidiendo un Goethe desde dentro. Carta a un alemán", en: Del mismo autor, Obras Completas, t. IV (1929-1933), 5ª ed., Madrid: Revista de Occidente, 1962, pp. 395-420; original publicado en abril de 1932.

ORTIZ, Luis, "Algunas consideraciones sobre la teoría de la acción finalista", en Revista de Ciencias Penales, t. XXIV (1965), pp. 4-24 (1 ${ }^{\mathrm{a}}$ parte) y pp.101-120 ( $2^{\mathrm{a}}$ parte); republicado en Londoño, Fernando; Maldonado, Francisco (Eds.), Clásicos de la literatura penal en Chile. La Revista de Ciencias Penales en el siglo XX: 1935-1995, 2 tomos, Valencia: Tirant Lo Blanch, 2018, t. II, pp. 11111136 ( $1^{\mathrm{a}}$ parte $)$ y pp. $1137-1160\left(2^{\mathrm{a}}\right.$ parte $)$.

ORTIZ, LUIS, "Presentación”, en: Londoño, Fernando; Maldonado, Francisco (Eds.), Clásicos de la literatura penal en Chile. La Revista de Ciencias Penales en el siglo XX: 1935-1995, 2 tomos, Valencia: Tirant Lo Blanch, 2018, t. I, pp. 23-26.

PAWLIK, Michael, La libertad institucionalizada. Estudios de Filosofía jurídica y Derecho penal, Madrid: Marcial Pons, 2010.

RAULD, Juan, "Ocho décadas de la Revista de Ciencias Penales y del Instituto de Ciencias Penales. 1935-2015: hitos y testimonios", en Londoño, Fernando; Maldonado, Francisco (Eds.), Clásicos de la literatura penal en Chile. La Revista de Ciencias Penales en el siglo XX: 1935-1995, 2 tomos, Valencia: Tirant Lo Blanch, 2018, t. I, pp. 27-50. 